\title{
Analysis of Handover Latency for Mobile IPv6 and mSCTP
}

\author{
Dong-Phil Kim* and Seok-Joo Koh*
}

\begin{abstract}
This paper analyzes the handover latency of Mobile IP and mobile SCTP over IPv6 networks. The analytical results are compared with the performance by experiment over Linux testbed. For analysis, we consider the two handover scenarios: horizontal handover and vertical handover. From the results, we see that mSCTP can provide smaller handover latency than Mobile IP. Moreover, mSCTP can give much smaller handover latency for vertical handover, compared to horizontal handover.
\end{abstract}

Keywords: Mobile IPv6, mSCTP, handover latency, vertical handover, horizontal handover

\section{Introduction}

At the time of the fixed-mobile convergence toward allIP networks, IP mobility has been focused in the nextgeneration wireless mobile networks that include WLAN and Cellular systems [1-3]. One of the essential issues for IP mobility is IP handover to provide a seamless handover for a Mobile Node (MN) that moves across different IP subnet regions while its sessions are active. The seamless handover can be realized by minimizing the handover latency and data losses.

Until now, several approaches have been proposed to support IP mobility. Among them, the Mobile IP (MIP) [45] has been regarded as a typical solution for IP handover in the network layer, whereas the mobile Stream Control Transmission Protocol (mSCTP) [6-8] was suggested for IP handover in the transport layer. In particular, the MIP can provide the location and handover management for terminal mobility in the network layer, whereas mSCTP can be used to support the end-to-end IP handover with the help of the multi-homing feature in the transport layer. In this paper, we will consider the MIPv6 rather than the Fast handover for MIPv6 (FMIPv6) [9] which is for further study.

In this paper, we analyze the handover latency of MIP and mSCTP over IPv6 networks theoretically with the two handover scenarios: single-homing $\mathrm{MN}$ for horizontal handover and dual-homing $\mathrm{MN}$ for vertical handover. We then compare the handover performance by experiment.

Manuscript received October 25, 2007; revised June 27, 2008; accepted August 25, 2008.

Corresponding Author: Seok-Joo Koh

* School of Electrical Engineering and Computer Science, Kyungpook

National University, Daegu, Korea (dpkim@cs.knu.ac.kr, sjkoh@knu.ac.kr)
For the experimental analysis, we construct a small test network based on the existing implementation codes for MIPv6. We also design and implement the mSCTP handover scheme, which allows the $\mathrm{MN}$ to automatically perform the mSCTP handover.

This paper is organized as follows. Section 2 and Section 3 describe the background and related works on MIP and mSCTP. In Section 4, we theoretically analyze the handover latency of MIP and mSCTP over IPv6 networks. Section 5 discusses the experimental results on MIP and mSCTP. Section 6 finally concludes this paper.

\section{Background}

In this section, we discuss the MIPv6 and mSCTP handover schemes briefly, and then compare the main inherent features of MIPv6 and mSCTP.

\subsection{MIPv6 Handover}

The MIPv6 has so far been regarded as a promising scheme for IP mobility in the network layer. In MIPv6, each $\mathrm{MN}$ is identified by its Home Address (HoA) made from the home network. While an $\mathrm{MN}$ is located away from its home network and moves into a new subnet region, it will acquire a care-of address (CoA) which provides information about its current point of attachment to the visited network. The MIPv6 is used by $\mathrm{MN}$ to register its new CoA with its Home Agent (HA).

In the MIPv6 handover, $\mathrm{MN}$ needs to detect the change of the subnet attached. To do this, MN can identify its movement into a new subnet by analyzing the Router Advertisement (RA) messages that are periodically sent by 
Access Router (AR). The MN might also achieve the movement detection by soliciting the RA message to the AR by sending a Router Solicitation (RS). After the movement detection, $\mathrm{MN}$ needs to acquire a new CoA by using the IPv6 address auto-configuration mechanism or DHCPv6. Once MN configures a new valid CoA, it must inform its newly configured address to HA by sending a Binding Update (BU) message which indicates the binding information between HoA and CoA. MN will then receive a Binding Acknowledgement (BA) message from HA. Note that MN in MIPv6 can send the BU and receive the BA to directly communicate with Correspondent Node $(\mathrm{CN})$, after completing the registration with the HA $[4,5]$.

\section{2 mSCTP Handover}

The mSCTP is defined as an SCTP protocol stack [6] with its ADDIP extension [7]. The mSCTP can be used to support IP handover for an $\mathrm{MN}$ in the transport layer. With mSCTP, each SCTP endpoint is able to add or delete an IP address to or from the existing association, and also to change the primary IP address for SCTP association.

Figure 1 illustrates the protocol operation of mSCTP for seamless handover in IPv6 networks.

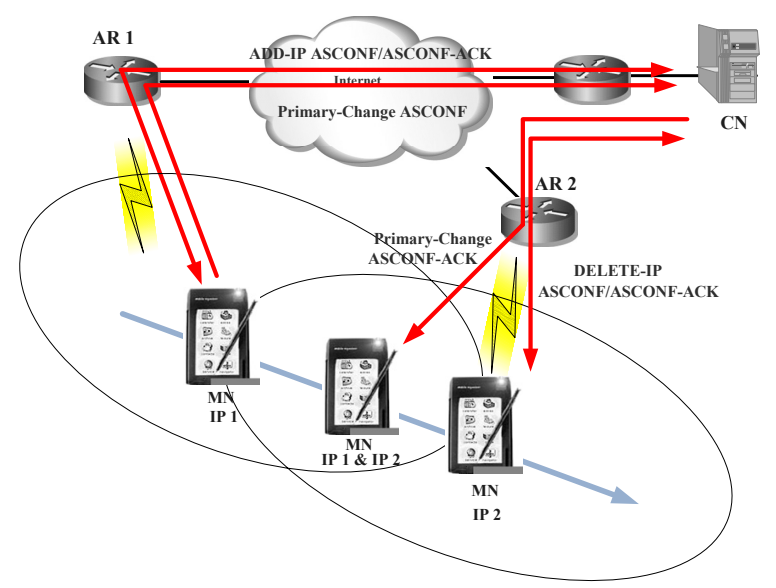

Fig. 1. mSCTP handover operations

In this figure, we assume that an MN initiates an SCTP association with $\mathrm{CN}$, and moves from AR1 to AR 2. For the association, $\mathrm{MN}$ initially uses 'IP address 1 ' in the AR1 region. Then, the overall $\mathrm{mSCTP}$ handover procedures could be performed as follows.

When an MN moves into the AR2 region, it detects the movement to a new subnet and obtains a new address 'IP address 2' by using the IPv6 address configuration scheme. After that, MN will inform the newly obtained IP address 2 to $\mathrm{CN}$. This is done by sending an SCTP Address Configuration (ASCONF) chunk to $\mathrm{CN}$. $\mathrm{MN}$ receives the responding ASCONF-ACK chunk from $\mathrm{CN}$. This is called by 'Add-IP' operation. After 'Add-IP' operation, the old IP address 1 is still used as the primary address, until the new IP address 2 is set to be the new 'Primary Address' by MN. Before the primary address is changed, IP address 2 is used for a backup path. As $\mathrm{MN}$ further continues to move toward AR 2, it needs to change the new IP address as its primary IP address. For this purpose, the MN sends the ASCONF chunk using the IP 1 address and receives the responding ASCONF-ACK chunk from $\mathrm{CN}$ over IP address 2 . Once the primary address is changed, $\mathrm{CN}$ sends the outgoing data packets to the new primary IP address of $\mathrm{MN}$. This is called 'Primary-Change' operation. As MN continues to move further toward AR2, it will delete the old IP address 1 from the association. This is called the 'Delete-IP' operation. These procedural steps will be repeated each time $\mathrm{MN}$ moves into a new subnet region.

\subsection{Comparison of MIPv6 and mSCTP}

Table 1 compares the basic inherent features of MIPv6 and $\mathrm{mSCTP}$.

Table 1. Comparison of MIPv6 and mSCTP

\begin{tabular}{c|c|c}
\hline Protocol layer & MIPv6 & mSCTP \\
\hline $\begin{array}{c}\text { Location } \\
\text { management }\end{array}$ & Provided & Not provided \\
\hline Mobility agents & HA layer & $\begin{array}{c}\text { No need of } \\
\text { mobility agents }\end{array}$ \\
\hline Route optimization & Need an extension & $\begin{array}{c}\text { Intrinsically } \\
\text { provided }\end{array}$ \\
\hline Handover support & $\begin{array}{c}\text { With the help of the } \\
\text { mobility agents }\end{array}$ & Provided \\
\hline
\end{tabular}

First of all, MIPv6 operates at the IP network layer to support the mobility. MIPv6 needs the route optimization extension to avoid the so-called triangular routing problem. Furthermore, MIP provides the location management but supports the limited handover with the help of the mobility agents such as HA or Foreign Agent (FA).

Secondly, mSCTP can be used to provide the seamless handover in the transport layer. mSCTP does not support the location management, but it can be used along with the MIP or SIP for location management $[10,11]$. On the other hand, the mSCTP does not require any additional mobility agents. It intrinsically provides the route optimization for data transport. 


\section{Related Works}

There have been several works [12-19] to investigate the performance of MIPv6 and mSCTP.

In terms of MIPv6, Lee et al. [12] analyzed the handover latency of MIPv6 by investigating the factors concerned with handover latency. They insist that the time taken for $\mathrm{MN}$ to detect its movement and address configuration is dominated into the main part of total Mobile IPv6 handover latency. Nicolas et al. [13] evaluated handover latency of MIPv6 over IEEE 802.11 wireless networks and compared the Layer 2 (L2) delays with Layer 3 (L3) delays. They concluded that the handover latency could be reduced considerably by using the anticipation of link layer trigger. On the other hand, Hernandez et al. [14] identified the limitations of MIP in terms of throughput, handover and packet loss at a different velocity of MN.

The study on mSCTP has also made recently. The work in [15] analyzes the mSCTP handover latency for vertical handover through some experiments. The work in [16] has analyzed that the handover latency with two different movement patterns (linear and cross movement pattern).

On the other hand, the work in [17] proposed mSCTP handover, called SIGMA, and compared handover latency of SIGMA and recent MIPv6 enhancements, considering the various experimental parameters. In [18], the authors analyzed and compared handover performance of MSCTP and MIP and Fast Handoff. In this work, it is concluded that while mSCTP handover gives less handover latency than MIP and Fast Handoff schemes, it may suffer from the packet reordering problem. The work in [19] argued that mSCTP handover provides better performance than MIP through the experimentation using the network simulator (NS-2). However, those works did not consider the dualhoming MN that an MN has two different wireless network interfaces.

This paper is purposed to compare the handover performance of MIPv6 and mSCTP by theoretical analysis as well as testbed experimentation. We will first analyze the handover latency of MIPv6 and MSCTP theoretically for the two movement scenarios: single-homing MN for horizontal handover and dual-homing $\mathrm{MN}$ for vertical handover. The analytical values will be compared and verified with the experimentation over Linux textbed. For experiment, we employed the recently released version of MIPv6 2.0.2 [20] and implemented the mSCTP handover.

\section{Analysis of Handover Latency}

In this section, we analyze the handover latency of MIP and mSCTP over IPv6 networks. For analysis, we consider the following two movement scenarios:

\section{$\underline{\text { Scenario A: Single-homing MN }}$}

The single-homing $\mathrm{MN}$ activates only a single network interface at a time. That is, MN performs the data transport in the single-homing state. This scenario could be applied to the horizontal handover, in which MN moves within a homogeneous network. In this case, the link-up of a new link and the link-down of the old link will occur at the same time.

\section{$\underline{\text { Scenario B: Dual-homing MN }}$}

The dual-homing MN uses the two different network interfaces in the overlapping region. That is, MN can transmit and receive data packets by using the two interfaces at the same time. This dual-homing MN could be applied to the vertical handover, in which MN moves across heterogeneous networks, as shown in the example of handover between $3 \mathrm{G}$ and WLAN.

In the analytical model, it is assumed that $\mathrm{MN}$ moves from an IP subnet to the other, and it has the ability to detect the link-up and link-down events with the help of the underlying link layer.

For the analytical purpose, we will take the following considerations for handover of an $\mathrm{MN}$ :

1) An MN is able to send an RS message as soon as the link-up event is detected;

2) Using the prefix information included in the RA message, an $\mathrm{MN}$ can configure its new CoA by using IPv6 stateless address auto-configuration mechanism;

3) Once an MN completes its tentative IPv6 address via the address auto-configuration mechanism, it proceeds with Duplicate Address Detection (DAD) process to confirm the validity of the IP address;

4) For DAD, only one Neighbor Solicitation (NS) message is enough to confirm the uniqueness of the tentative IPv6 address;

5) For MIPv6, the Return Routability (RR) procedure is used to support a secure communication between $\mathrm{MN}$ and $\mathrm{CN}$. It is assumed that the RR procedure 
will be done just after completing BU to HA [5];

6) For mSCTP, the SCTP ASCONF chunks that are exchanged between the two endpoints. For secure signaling, those chunks are encapsulated using an appropriate Authentication (AUTH) chunks.

In particular, we make the following assumptions for handover of a dual-homing $\mathrm{MN}$ in the overlapping region:

1) The overlapping region between different subnets is enough large, and the sojourn time of $\mathrm{MN}$ in this region is larger than the time taken to perform the ADD-IP operation of mSCTP.

2) $\mathrm{MN}$ can use two IP addresses in the overlapping region.

Based on the assumptions made above, we will analyze the handover latency of MIPv6 and mSCTP.

\subsection{Mobile IPv6 Handover Latency}

Fig. 2 depicts the overall latency of MIPv6 handover.

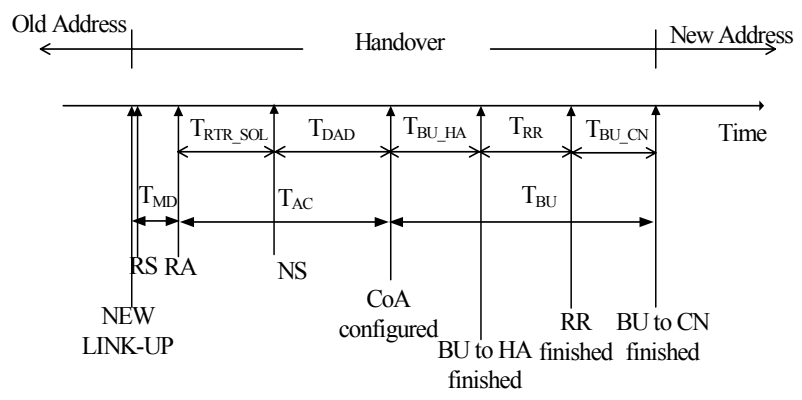

Fig. 2. MIP handover latency for the single-homing $\mathrm{MN}$

As shown in the figure, the overall MIPv6 handover latency for the single-homing $\mathrm{MN}$ can be calculated as:

$$
T_{\text {Handover_MIP }}=T_{M D}+T_{A C}+T_{B U}
$$

In the equation, $T_{M D}$ is Movement Detection (MD) delay, $T_{A C}$ is Address Configuration (AC) delay, and $T_{B U}$ is BU delay. The MD delay is the time taken for $\mathrm{MN}$ to detect its movement into a new subnet. The AC delay is the time required for $\mathrm{MN}$ to configure a new $\mathrm{CoA}$ using the prefix information contained in the RA message. The BU delay is the time taken for $\mathrm{MN}$ to register the new CoA with HA, to perform the RR procedure, and then to register with $\mathrm{CN}$.

It is noted that $T_{M D}$ could further be divided into the $\mathrm{L} 2$ handover delay and L3 MD delay. The L2 handover delay depends on a specific link layer technology and may be negligible compared to L3 MD delay. So, we will focus on L3 MD delay in this paper. The L3 MD delay might get larger in the network, where $\mathrm{MN}$ relies on RAs for movement detection. However, if an $\mathrm{MN}$ is able to send an $\mathrm{RS}$ as soon as it receives an L2 trigger from the lower layer, the MD delay will also be reduced.

After the movement detection, an $\mathrm{MN}$ begins to configure a new $\mathrm{CoA}$ and performs the DAD process to confirm the uniqueness of the CoA address on the subnet. The $T_{A C}$ can be calculated by summing up the time taken for random waiting delay of an NS message $\left(T_{R T R} S O L\right)$ and for the DAD delay to wait a feedback from a neighboring node in the subnet $\left(T_{D A D}\right)$.

On completion of a new CoA configuration, $\mathrm{MN}$ needs to register the CoA with $\mathrm{HA}$ and $\mathrm{CN}$. For this purpose, the $\mathrm{MN}$ will perform the RR procedure to ensure the secure communication between $\mathrm{MN}$ and $\mathrm{CN}$. Accordingly, the BU delay can be expressed as follows:

$$
T_{B U}=T_{B U-H A}+T_{R R}+T_{B U-C N}
$$

Let us denote by $D_{X \cdot Y}$ the one way transmission delay between two nodes $X$ and $Y$. Then, $T_{B U-H A}=D_{M N \cdot H A}+$ $D_{H A \cdot M N}, T_{R R}=\max \left[\left(D_{M N \cdot H A}+D_{H A \cdot C N}\right), D_{M N \cdot C N}\right]+\max$ $\left[\left(D_{C N \cdot H A}+D_{H A \cdot M N}\right), D_{C N \cdot M N}\right]=D_{M N \cdot H A}+D_{H A \cdot C N}+D_{C N \cdot H A}+$ $D_{H A \cdot M N}\left(\right.$ if $\left.D_{M N \cdot H A}+D_{H A \cdot C N}>D_{M N \cdot C N}\right)$, and $T_{B U-C N}=D_{M N \cdot C N}+$ $D_{C N \cdot M N}$.

By assumption of $D_{X \cdot Y}=D_{Y \cdot X}$, we get $T_{B U-H A}=2 D_{M N \cdot H A}$, $T_{R R}=2 \cdot\left(D_{M N \cdot H A}+D_{H A \cdot C N}\right)$, and $T_{B U-C N}=2 \cdot D_{M N \cdot C N}$.

Accordingly, the total BU delay is rewritten as

$$
T_{B U}=2 \cdot\left(2 \cdot D_{M N \cdot H A}+D_{H A \cdot C N}+D_{M N \cdot C N}\right)
$$

In summary, the overall MIPv6 handover delay for the single-homing MN, Equation (1) can be expressed as

$$
\begin{aligned}
T_{\text {Handover_MIP }}= & T_{M D}+T_{A C}+ \\
& 2 \cdot\left(2 \cdot D_{M N \cdot H A}+D_{H \cdot A \cdot C N}+D_{M N \cdot C N}\right)
\end{aligned}
$$

On the other hand, Fig. 3 depicts the handover latency of MIPv6 for the dual-homing MN.

For the dual-homing $\mathrm{MN}, \mathrm{MN}$ can still communicate with $\mathrm{CN}$ during $\mathrm{MD}$ and $\mathrm{AC}$ operations, which is different from the single-homing MN. Accordingly, the MIPv6 handover latency can be reduced to $T_{B U}$, as shown in Fig. 3 . That is, the overall MIPv6 handover latency for the dualhoming $\mathrm{MN}$ can be summarized as Equation (5).

$$
T_{\text {Handover_MIP }_{-}}=T_{B U}=2 \cdot\left(2 \cdot D_{H A \cdot M N}+D_{H A \cdot C N}+D_{M N \cdot C N}\right)
$$




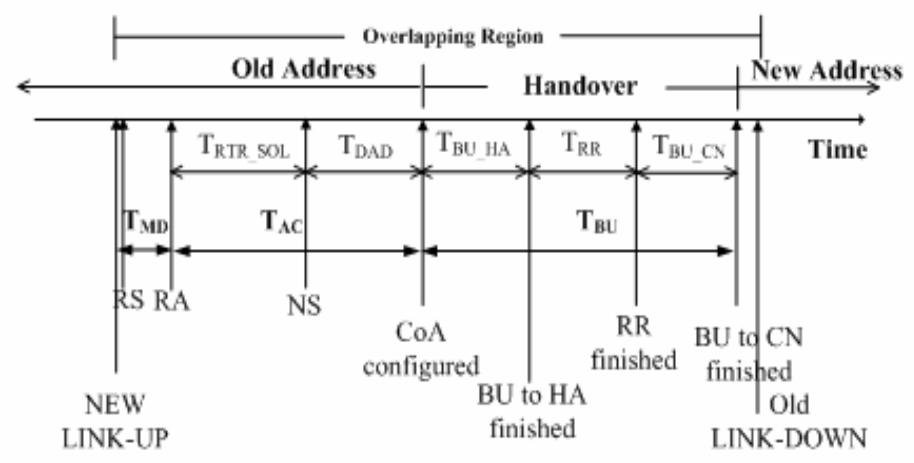

Fig. 3. MIP handover latency for the dual-homing MN

\section{2 mSCTP Handover Latency}

In the mSCTP handover, the handover performance could depend on how to configure the triggering rules for ADD-IP and Primary-Change operations. Some works have so far been made to deal with this issue. The work in [21] insists that the ADD-IP and Primary-Change operations should be done by comparing the link signal strength information. The work in [22] proposed a scheme performing the Primary-Change operation by comparing the network bandwidth and round trip time in the concerned networks. The scheme proposed in [23] is to perform the Primary-Change operation when RTT of the candidate path is smaller than that of the current primary path. Most of the existing works insist that the Add-IP and Primary-Change operations should be done if a new path has a better condition than the existing primary path.

In this paper, we consider the following triggering rules for Add-IP and Primary-Change operations. The singlehoming MN performs the Add-IP and Primary-Change operations at the same time, as soon as it enters a new subnet. For the dual-homing MN, the Add-IP operation will be done just after MN moves into a new subnet, which will be detected by a Link-Up trigger. Then, the Primary-
Change operation will be triggered after the L2 handover has been completed.

In the meantime, it is assumed that mSCTP uses the identical MN and AC schemes with MIPv6. Now, denote $T_{m S C T P}$ by the time taken for the MSCTP operations to exchange the SCTP ASCONF and ASCONF-ACK chunks between $\mathrm{MN}$ and $\mathrm{CN}$.

Then, the overall handover latency of mSCTP can be expressed as:

$$
T_{\text {Handover_ } m \text { } m S C T P}=T_{M D}+T_{A C}+T_{m S C T P}
$$

Fig. 4 depicts the delay components associated with the mSCTP handover latency for the single-homing MN.

In the single-homing $\mathrm{MN}$, the mSCTP handover delay includes the time for MN to perform the Add-IP and Primary-Change operations. That is, the mSCTP handover delay corresponds to the transmission delays taken to exchange the ASCONF and ASCONF-ACK chunks between $\mathrm{MN}$ and $\mathrm{CN}$, as shown in Equation (7).

$$
T_{m S C T P}=T_{A D D-I P}+T_{P-C}=2 \cdot\left(D_{M N \cdot C N}+D_{C N \cdot M N}\right)
$$

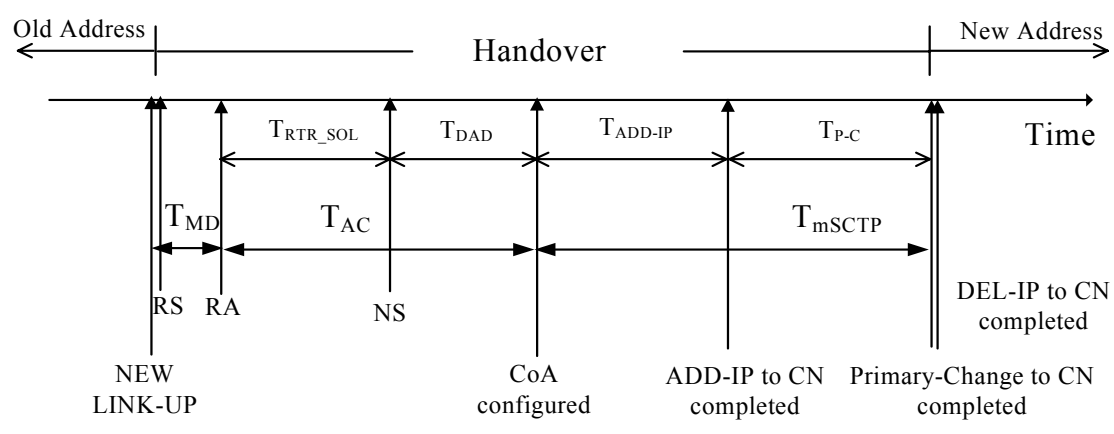

Fig. 4. mSCTP handover latency for single-homing MN 


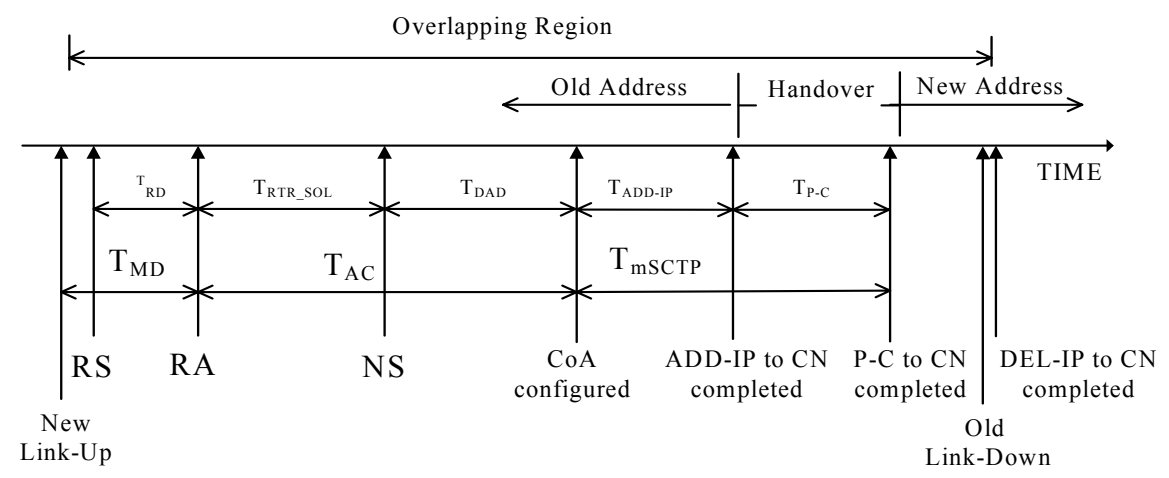

Fig. 5. mSCTP handover latency for dual-homing MN

In the equation, it is noted that $T_{A D D-I P}=D_{M N \cdot C N}+D_{C N \cdot M N}$ and $T_{P-C}=D_{M N \cdot C N}+D_{C N \cdot M N}$. In summary, the overall $\mathrm{mSCTP}$ handover latency can be summarized as follows:

$$
T_{\text {Handover_mSCTP }}=T_{M D}+T_{A C}+4 \cdot D_{M N \cdot C N}
$$

Fig. 5 analyzes the overall mSCTP handover latency for the dual-homing MN.

In the dual-homing $\mathrm{MN}$, the total mSCTP handover latency includes only the time taken for $\mathrm{MN}$ to perform the Primary-Change operation, because $\mathrm{MN}$ can communicate with $\mathrm{CN}$ during the $\mathrm{MD}$ and $\mathrm{AC}$ and Add-IP operations, as shown in Fig. 5.

Accordingly, the overall latency of mSCTP handover for the dual-homing MN can be summarized as follows:

$$
T_{\text {Handover_mSCTP }}=2 \cdot D_{M N \cdot C N}
$$

\subsection{Comparison of mSCTP and MIPv6}

Table 2 compares the handover latency for mSCTP and MIPv6.

\begin{tabular}{|c|c|c|}
\hline & MIPv6 & mSCTP \\
\hline $\begin{array}{l}\text { Handover latency } \\
\text { for single-homing }\end{array}$ & $\begin{array}{c}T_{M D}+T_{A C}+2 \cdot\left(2 \cdot D_{M N-}\right. \\
\left.{ }_{H A}+D_{H A \cdot C N}+D_{M N-C N}\right)\end{array}$ & $\begin{array}{c}T_{M D}+T_{A C}+ \\
4 \cdot D_{M N \cdot C N}\end{array}$ \\
\hline $\begin{array}{l}\text { Handover latency } \\
\text { for dual-homing }\end{array}$ & $2 \cdot\left(2 \cdot D_{M N \cdot H A}+D_{H A \cdot C N}+D_{M N \cdot C N}\right)$ & $2 \cdot D_{M N \cdot C N}$ \\
\hline
\end{tabular}

Table 2. Handover latency for MIPv6 and mSCTP

From the table, we can see that MIPv6 gives larger handover latency than mSCTP for both the cases, about by $2 \cdot\left(2 \cdot D_{M N \cdot H A}+D_{H A \cdot C N}\right)$, which corresponds to the BU delays of MIPv6 through HA (i.e., BU between HA and $\mathrm{CN}$, and BU between HA and MN). This is because the MIPv6 operations are dependent on the binding update with HA.

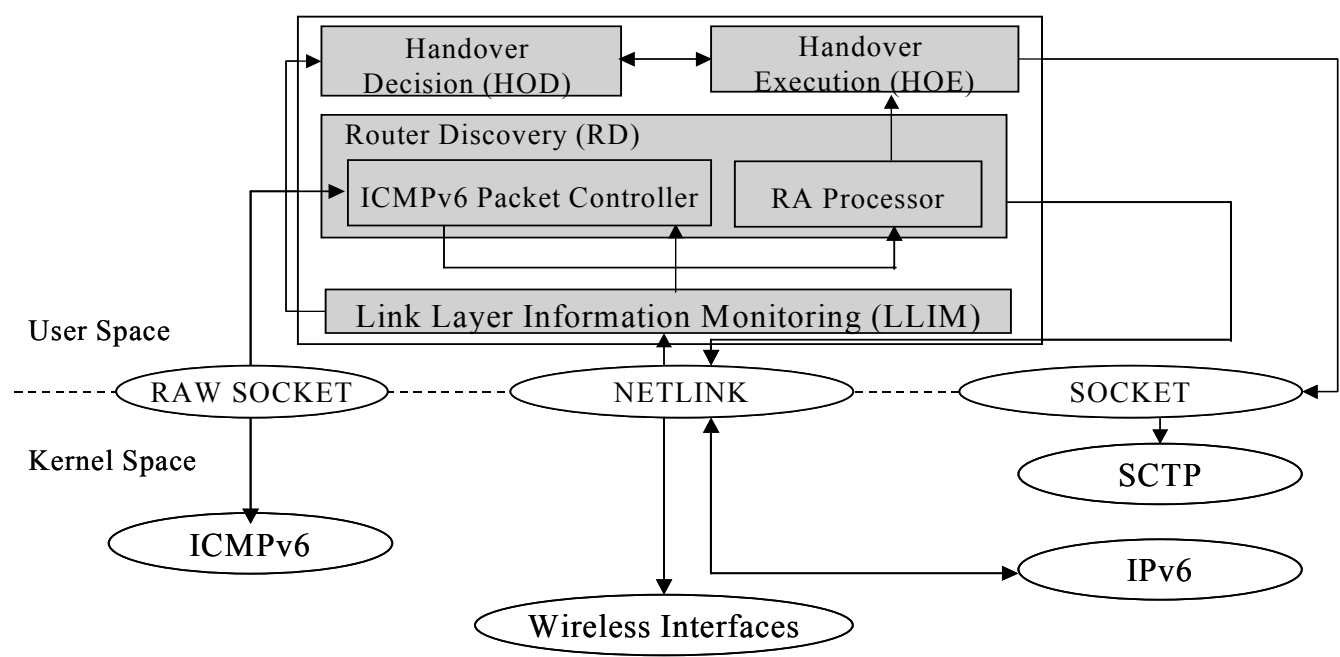

Fig. 6. mSCTP handover supporting system 


\section{Experimental Results}

This section describes the experimental results for MIPv6 and mSCTP that are performed over Linux testbed.

\subsection{Test Environment}

To perform the experiments, we construct a small test network where consists of a router and two hosts (MN and $\mathrm{CN}$ ). In addition, $\mathrm{HA}$ is installed for experimentation of MIPv6. For MIPv6, we installed the open source codes of MIPL 2.0.2 [20]. On the other hand, for experimentation of mSCTP handover, we implemented the associated system that enables $\mathrm{MN}$ to automatically support mSCTP handover in the IPv6 networks.

Fig. 6 shows the implementation architecture of mSCTP handover supporting system.

As shown in the figure, the MSCTP handover supporting system consists of four modules: Link Layer Information Monitoring (LLIM), Router Discovery (RD), Handover Decision (HOD), and Handover Execution (HOE). LLIM is used to gather the link layer information of wireless interfaces to achieve faster movement detection. $\mathrm{RD}$ is used to discover the new AR in the new subnet region. HOD is used to determine when to perform the PrimaryChange operation using the received signal strengths. HOE is used to execute the MSCTP handover operations, which include the exchange of mSCTP ASCONF and ASCONFACK chunks.

LLIM gathers the link information of wireless interfaces concerned with $\mathrm{MN}$ including the link-up/down and signal strength information. Such information could be generated by the kernel wireless subsystem. To utilize LLIM, we modified the existing codes of wireless tools [24] to monitor the wireless events generated from the designated wireless interfaces.

If a link-up event is detected from LLIM, RD module tries to discover the new AR in the new subnet. For this purpose, RD is constructed as ICMPv6 packet controller and RA processor. The ICMPv6 packet controller sends the RS messages via the ICMPv6 raw socket API and receives the solicited RA message as a response. RA processor then compares the prefix information of the received RA messages with the current ongoing prefix. If the received prefix information is a new one, $\mathrm{RD}$ recognizes that $\mathrm{MN}$ enters the new subnet and then requests ADD-IP operation to the HOE module.

HOE initially initiates the SCTP association with CN. After that, if the ADD-IP operation is required by RD,
HOE sends an ASCONF to $\mathrm{CN}$ by calling the sctp_bindx() function. It then processes the corresponding ASCONFACK chunk. Moreover, when the Primary-Change operation is called by HOD, HOE sends an ASCONF chunk for changing the primary path by calling the setsockopt() function.

On the other hand, HOD module determines when to perform the Primary-Change operation by comparing the received signal strengths from LLIM. To perform this, HOD periodically stores the signal strength information received from LLIM and then compares them. If the signal strength of the new alternate path is larger than the primary path, HOD requests the Primary-Change operation to HOE.

Fig. 7 shows the network topology of the test network. The test network has three subnets: two wireless networks (AR1 and AR2) with an IEEE 802.11 Access Point (AP) and one wired network (AR3) based on 100Mbps Ethernet. MIPv6 HA is embedded at AR1. Thus, the one-way transmission delay between MN and HA gets larger as an MN moves further toward the AR2 region. Moreover, it is noted that the one-way transmission delay between MN and $\mathrm{CN}$ through HA is larger than that between MN and $\mathrm{CN}$. In the test network, MN is initially connected to AP1 of AR1. At the 20s after the experiment, we enforced the $\mathrm{MN}$ to perform L2 handover to AP2 of AR2.

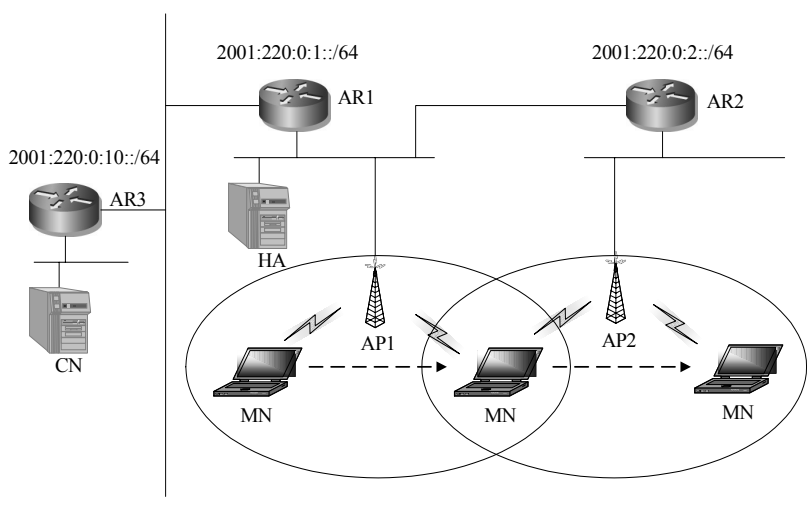

Fig. 7. Test network

\subsection{Results and Discussion}

Table 3 and 4 summarize the experimental results for MIPv6 and mSCTP in terms of the handover delay components. In the tables, the MD delay is corresponded to L3 MD delay. Based on the numerical values measured from experiment, we calculate the total handover latency for the single-homing MN by using Equation (4) and (8), and the total handover latency for the dual-homing $\mathrm{MN}$ by using Equation (5) and (9). 
Table 3. Handover latency for MIPv6

\begin{tabular}{c|c|c|c|c|c}
\hline & $T_{M D}$ & $T_{A C}$ & $T_{B U-H A}$ & $T_{R R}$ & $T_{B U-C N}$ \\
\hline Min & 0.053 & 1.019 & 0.812 & 0.698 & 0.853 \\
\hline Max & 0.097 & 1.982 & 1.006 & 0.939 & 0.914 \\
\hline Avg. & 0.075 & 1.501 & 0.909 & 0.819 & 0.884 \\
\hline & \multicolumn{2}{|c|}{$T_{\text {Handover_MIPv6 }}$ for Single-homing MN } & \multicolumn{3}{|c}{$T_{\text {Handover_MIPv6 }}$ for Dual-homing MN } \\
\hline Min & \multicolumn{3}{|c|}{3.435} & \multicolumn{3}{|c}{2.363} \\
\hline Max & \multicolumn{3}{|c|}{4.938} & \multicolumn{3}{|c}{2.659} \\
\hline Avg. & \multicolumn{3}{|c|}{4.188} & & \multicolumn{3}{|c}{} \\
\hline
\end{tabular}

Table 4. Handover latency for mSCTP

\begin{tabular}{c|c|c|c|c}
\hline & $T_{M D}$ & $T_{A C}$ & $T_{A D D-I P}$ & $T_{P-C}$ \\
\hline Min & 0.028 & 1.012 & 0.165 & 0.293 \\
\hline Max & 0.102 & 1.744 & 0.303 & 0.507 \\
\hline Avg. & 0.065 & 1.378 & 0.234 & 0.400 \\
\hline & \multicolumn{2}{|c|}{$T_{\text {Handover_mSCTP: }}$} & \multicolumn{2}{|c}{$T_{\text {Handover_mSCTP }}$} \\
& for Single-homing MN & \multicolumn{2}{c}{ for Dual-homing MN } \\
\hline Min & \multicolumn{2}{|c|}{1.498} & \multicolumn{2}{c}{0.293} \\
\hline Max & \multicolumn{2}{|c|}{2.656} & \multicolumn{2}{c}{0.507} \\
\hline Avg. & \multicolumn{2}{|c|}{2.077} & \multicolumn{2}{c}{0.400} \\
\hline
\end{tabular}

In Table 3 , it is noted that the $T_{R R}$ is almost similar to $T_{B U-C N}$ on the average. However, $T_{R R}$ is greater than $T_{B U-C N}$ on the minimum value, whereas $T_{B U-C N}$ is greater than $T_{R R}$ in the maximum value. These unexpected results seem to occur because in the MIPv6 implementation code [20] (used for our experiment), the processing time of $\mathrm{CN}$ and/or $\mathrm{MN}$ to handle the $\mathrm{BU} / \mathrm{BA}$ messages tends to be too much irregular than expected, depending on the data traffics at $\mathrm{CN}$ and/or $\mathrm{MN}$ during experiment.

From the overall results, we can see that mSCTP handover latency for the single-homing $\mathrm{MN}$ is smaller than MIPv6 handover latency approximately by 2 second. This is mainly because the MIPv6 BU delays between HA and $\mathrm{MN}$ are relatively large, compared to the delays taken for the mSCTP ADD-IP and Primary-Change operations. It is noted that the ADD-IP and Primary-Change operations are completed within $300 \mathrm{~ms}$.

In case of the dual-homing $\mathrm{MN}$, it is also shown that the mSCTP gives smaller handover latency than MIPv6. This is because mSCTP handover needs the delay taken for the ADD-IP and Primary-Change operations, whereas MIPv6 requires much longer handover latency for $\mathrm{BU}$ operations with $\mathrm{HA}$ and $\mathrm{CN}$.

Moreover, it is noted that the dual-homing MN (for both of mSCTP and MIPv6) tends to give much smaller handover latency than the single-homing $\mathrm{MN}$. This is because the data packet delivery between $\mathrm{MN}$ and $\mathrm{CN}$ can be made during the handover operation in the dual-homing $\mathrm{MN}$, differently from the single-homing $\mathrm{MN}$.
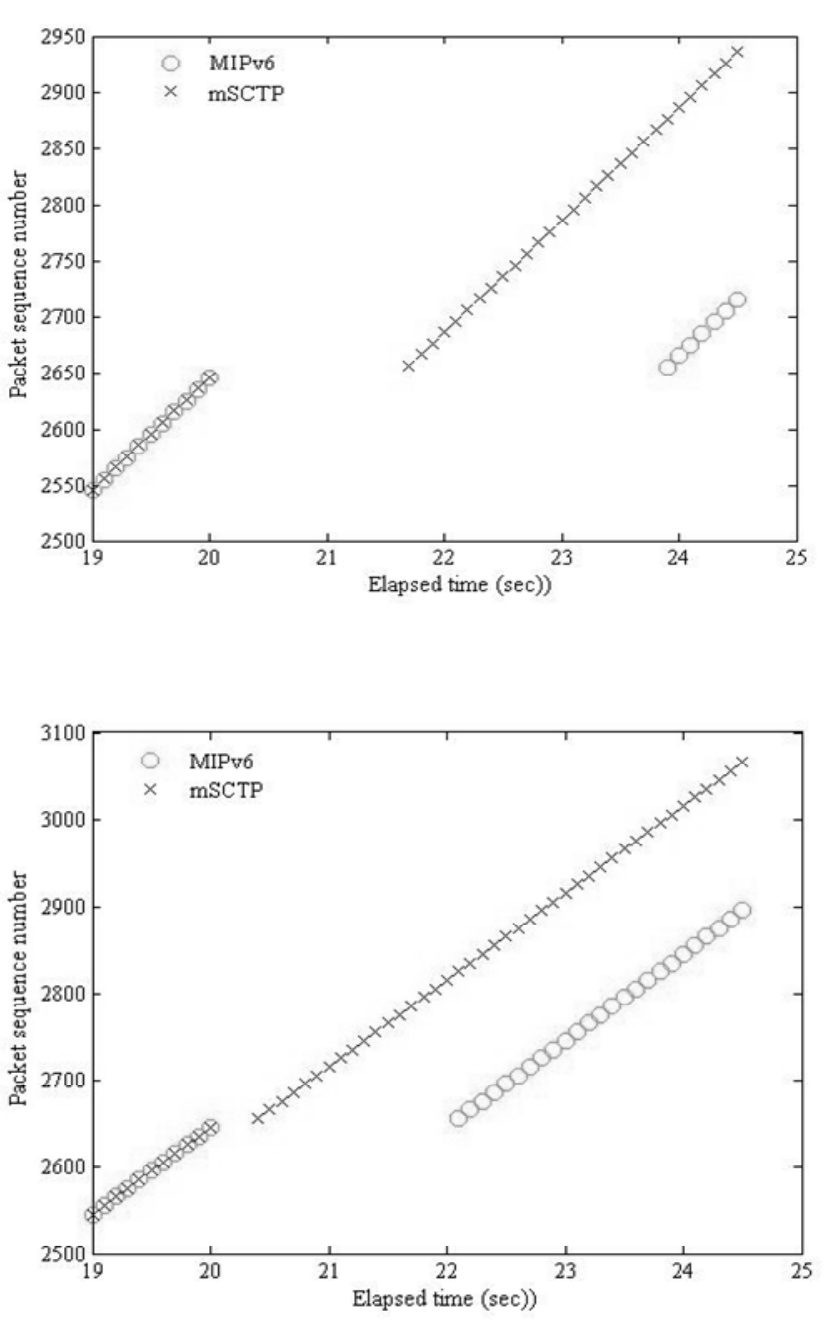

Fig. 8. Throughputs for MIPv6 and mSCTP by handover

From the results, we can also see that these empirical results are nearly similar to the theoretical analysis that was described in Section 4.

Fig. 8 plots the sequence numbers of data packets transmitted for comparison of throughputs during handover. For experiment, we enforced $\mathrm{MN}$ to perform the L2 handover from $\mathrm{AP} 1$ of $\mathrm{AR} 1$ to $\mathrm{AP} 2$ of $\mathrm{AR} 2$ region at the time of $20 \mathrm{~s}$.

From the figure, it is shown that mSCTP provides better throughput performance than MIPv6. We can also see that the dual-homing MN scenario gives smaller delay than the single-homing MN scenario. It is noted that MIPv6 gives larger handover latency than mSCTP approximately by 2 seconds. These results are almost consistent with those in Table 3 and 4. 


\section{Conclusion}

In this paper, we have compared the handover latency of MIP and mSCTP over IPv6 networks for the two handover scenarios: single-homing $\mathrm{MN}$ for horizontal handover and dual-homing $\mathrm{MN}$ for vertical handover. We first analyzed the handover latency for the two schemes theoretically by investigating the delay components associated with handover. We then compare the handover performance of MIPv6 and mSCTP over a Linux-based testbed by experimentation.

For the analytical and experimental results, we can see that mSCTP gives smaller handover latency than MIPv6 for the single-homing and dual-homing handover scenarios. In particular, in the dual-homing MN case, mSCTP gives smaller handover latency than MIPv6. This is because mSCTP needs the delay taken for ADD-IP and PrimaryChange operations, whereas MIPv6 requires much longer handover latency for BU operations with $\mathrm{HA}$ and $\mathrm{CN}$.

It is noted that the dual-homing $\mathrm{MN}$ tends to give much smaller handover latency than the single-homing MN. This is because the data packet delivery between $\mathrm{MN}$ and $\mathrm{CN}$ can be made during handover in the dual-homing MN. We also note that the empirical results are almost consistent with the theoretical analysis.

\section{Acknowledgement}

This research was supported by the MKE (The Ministry of Knowledge Economy) of Korea, under the ITRC (Information Technology Research Center) support program supervised by the IITA (Institute for Information Technology Advancement): (IITA-2008-C1090-08040004).

\section{Reference}

[1] Lin Y. et al., Wireless and Mobile Network Architecture, John Wiley \& Sons, Inc., 2001.

[2] Evans B. G. and McLaughlin S., "Visions of 4G," IEE Electronics \& Communication Engineering Journal, December 2000.

[3] Ala-Laurila J. et al., "Wireless LAN Access Network Architecture for Mobile Operators," IEEE Communication Magazine November 2001.

[4] Perkins, C., et al., IP Mobility Support for IPv4,
IETF RFC 3344, August 2002.

[5] Johnson, D., et al., Mobility Support in IPv6, IETF RFC 3775, June 2004.

[6] Stewart R., et al., Stream Control Transmission Protocol, IETF RFC 2960, October 2000.

[7] Stewart, R., et al., Stream Control Transmission Protocol (SCTP) Dynamic Address Reconfiguration, IETF RFC 5061, September 2007.

[8] Koh S., et al, "mSCTP for Soft Handover in Transport Layer," IEEE Communications Letters, Vol. 8, No.3, pp. 189-191, March 2004.

[9] Koodli R, et al., Fast Handovers for Mobile IPv6, IETF RFC 4068, July 2005.

[10] Koh S., et al., Mobile SCTP with Mobile IP for Transport Layer Mobility, IETF Internet Draft, June 2004.

[11] Aydin I., et al., "Cellular SCTP: A Transport-Layer Approach to Internet Mobility," Proceeding of ICCCN 2003, pp. 285-290, October 2003.

[12] Lee S., et al., "Analysis of Handoff Delay for Mobile IPv6," Proc. of VTC 2004, pp. 26-29, September 2004.

[13] Nicolas, M., et al, "Analysis and Evaluation of Mobile IPv6 Handovers over Wireless LAN," Mobile Networks and Applications, Vol. 8, No. 6, pp. 643653, December 2003.

[14] Hernandez E., et al., "Examining Mobile IP Performance in Rapidly Mobile Environments: The Case of a Commuter Train," Proc. of 26th IEEE Local Computer Networks (LCN'01), pp. 365-372, Feb., 2001.

[15] Koh S., et al., "mSCTP for Vertical Handover between Heterogeneous Networks," LNCS 3597, pp. 28-36, July 2005.

[16] Kim D., et al. "Analysis of SCTP Handover by Movement Patterns," LNCS 3645, Vol. 3545, pp. 521-529, August 2005.

[17] Sivaqurunathan S., et al., "Experimental Comparison of Handoff Performance of SIGMA and Mobile IP," Proc. of High Performance Switching and Routing, pp. 366-370, May 2005.

[18] Ken C. K., et al., "Handoff Performance Comparison of Mobile IP, Fast Handoff and mSCTP in Mobile Wireless Networks," Proc. of I-SPAN 2008, pp. 4552, May 2008.

[19] Antonios A., et al., "A Soft-handoff Transport Protocol for Media Flows in Heterogeneous Mobile Networks," Computer Networks, Vol. 50, Issue. 11, pp. 1860-1871, August 2006. 
[20] MIPL Mobile IPv6 for Linux, Available from http://www.mipl.mediapoli.com.

[21] Chang M., et al., "Transport Layer Mobility Support Utilizing Link Signal Strength Information,” IEICE Transactions on Communications, Vol. E87-B, No. 9, pp. 2548-2556, September 2004.

[22] Junichi, F., et al., "Study on Primary Path Switching Strategy," Workshop on Cooperative Computing, in Proc. of WCCIA 2005, pp. 536-541, April 2005.

[23] Kim D., et al., "Adaptive Primary Path Switching for SCTP Handover," Proc. of ICACT 2008, Vol. 2, pp. 900-902, February 2008.

[24] Wireless Tools for Linux, Available from http://www.hpl.hp.com/personal/Jean_Tourrilhes/Lin ux

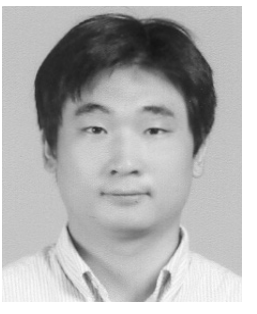

\section{Dong-Phil Kim}

He received B.S. degree in Computer Aided Science from Inje University, Korea in 2003. He received M.S. degree in Department of Information Security from the Kyungpook National University. He is taking Ph.D. course in Department of Information Security from Kyungpook National University. His current research interests include Internet Mobility, mobile SCTP.

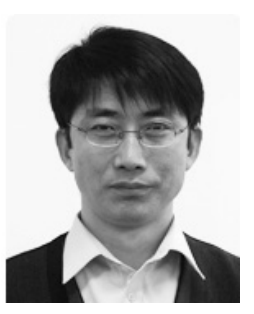

\section{Seok-Joo Koh}

He received B.S. and M.S. degrees in Management Science from KAIST in 1992 and 1994, respectively. He also received Ph.D. degree in Industrial Engineering from KAIST in 1998. From August 1998 to February 2004, he worked for Protocol Engineering Center in ETRI. He is now an Assistant Professor at Electronics Engineering and Computer Science in the Kyungpook National University since March 2004. His current research interests include Mobility Management in SBI2K, Internet Mobility, Multicasting, and SCTP. He has so far participated in the International Standardization as an editor in ITU-T SG19, SG17, SG13, ISO/IEC JTC1/SC6, and IETF. 